\title{
No transfer of calibration between action and perception in learning a golf putting task
}

\author{
Wim Van Lier • John Van der Kamp • \\ Anne van der Zanden • Geert J. P. Savelsbergh
}

Published online: 4 August 2011

(C) The Author(s) 2011. This article is published with open access at Springerlink.com

\begin{abstract}
We assessed calibration of perception and action in the context of a golf putting task. Previous research has shown that right-handed novice golfers make rightward errors both in the perception of the perfect aiming line from the ball to the hole and in the putting action. Right-handed experts, however, produce accurate putting actions but tend to make leftward errors in perception. In two experiments, we examined whether these skill-related differences in directional error reflect transfer of calibration from action to perception. In the main experiment, three groups of righthanded novice participants followed a pretest, practice, posttest, retention test design. During the tests, directional
\end{abstract}

The authors thank Berry den Brinker for his help in data analysis.

W. Van Lier $\cdot$ J. Van der Kamp $\cdot$ A. van der Zanden •

G. J. P. Savelsbergh

Research Institute Move, Faculty of Human Movement Sciences,

VU University, Amsterdam, The Netherlands

W. Van Lier · G. J. P. Savelsbergh

Academy for Physical Education,

University of Professional Education,

Amsterdam, The Netherlands

J. Van der Kamp

Institute of Human Performance, University of Hong Kong,

Hong Kong, Hong Kong SAR

G. J. P. Savelsbergh

Research Institute for Biomedical Research into Human

Movement and Health, Faculty of Science and Engineering,

Manchester Metropolitan University,

Manchester, UK

W. Van Lier $(\triangle)$

Faculty of Human Movement Sciences, VU University,

Van der Boechorststraat 9,

NL 1081 BT Amsterdam, The Netherlands

e-mail:w.h.van.lier@vu.nl error for the putting action and the perception of the perfect aiming line were determined. During practice, participants were provided only with verbal outcome feedback about directional error; one group trained perception and the second trained action, whereas the third group did not practice. Practice led to a relatively permanent annihilation of directional error, but these improvements in accuracy were specific to the trained task. Hence, no transfer of calibration occurred between perception and action. The findings are discussed within the two-visual-system model for perception and action, and implications for perceptual learning in action are raised.

Keywords Perceptual learning · Far aiming · Calibration . Two-visual-system model $\cdot$ Transfer

\section{Introduction}

In putting, right-handed novice golfers who stand to the left side of the ball make systematic directional errors to the right of the hole (Johnston, Benton \& Nishida, 2003; Roberts \& Turnbull, 2010; Van Lier, Van der Kamp \& Savelsbergh, 2011). These putting errors by novice golfers appear to be predicated on the misperception of the direction of the perfect aim line between the ball and the hole, although the rightward errors in perception are smaller than the rightward putting errors. Intriguingly, although skilled golfers aim accurately when putting, they tend to make leftward errors in the perception of the perfect aim line (see Fig. 1). Van Lier et al. argued that skilled golfers had managed to overcome the rightward errors by a process of calibration, during which they presumably adjusted the relation between directional information and the control of the orientation of the club head relative to ball. They further 
Fig. 1 Directional errors in the putting action (dashed lines) and the perception of the perfect aiming line (plain lines) for novice and skilled golfers

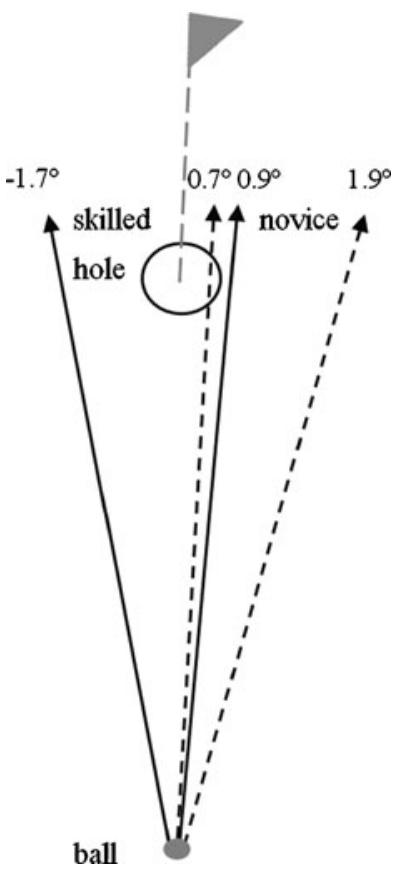

proposed that the parallel leftward shift in perception may suggest that this calibration of putting action transferred to the perception of direction. The nature of a learning process, however, cannot be conclusively inferred from performance differences between groups of different skill levels. In addition, it cannot be ruled out that the skilled participants in the study by Van Lier et al. had first calibrated the perception of direction and subsequently transferred this calibration into the putting action. In order to address these issues, the present study employed a learning experiment during which novice golfers learned to overcome the directional errors in the perception of the perfect aim line or in the putting action by practicing either the perceptual judgments or the putting action.

The first purpose of this study was to verify whether learning to aim a golf putt indeed is consistent with a process of calibration. From an ecological approach, calibration is defined as the scaling or adjustment of an action or perceptual judgment to the information that is used. That is, proponents of the ecological approach argue that for any task, a lawful relation exists between information (i.e., $I_{(\mathrm{t})}$ ) and task (i.e., $T_{(\mathrm{t})}$ ) variables (Warren, 1988; see also Van der Kamp, Oudejans \& Savelsbergh, 2003). In its simplest appearance, this so-called law of control is formally expressed as

$T_{(\mathrm{t})}=a+b^{*} I_{(\mathrm{t})}$,

in which $T_{(\mathrm{t})}$ stands for a particular task variable (e.g., in the case of putting, the orientation of the club head at impact, or in the case of a perceptual judgment, the perception of the direction of a line), $I_{(\mathrm{t})}$ stands for a particular information variable (e.g., an optic variable specifying the direction toward the hole), and $a$ and $b$ stand for constants that reflect the precise relationship between the task and information variables. Calibration refers to a change in the relation between the task and optic variables by tuning of the constants $a$ and $b$ (Jacobs \& Michaels, 2007; Withagen $\&$ Michaels, 2005; for an alternative theoretical account of calibration, see, e.g., Ernst \& Banks, 2002).

A popular paradigm for examining the process of calibration has been the use of wedge prisms (e.g., Redding \& Wallace, 1997). By laterally displacing the field of view, wedge prisms artificially manipulate the relation between informational and task variables. Martin, Keating, Goodkin, Bastian and Thach (1996), for example, examined participants' adjustments in throwing direction after donning prisms. Initially, participants made large directional errors in line with the artificial lateral displacement, but they rapidly adapted to the optical distortion. The directional errors had been reduced to zero within 10 to 30 throws. After removal of the prisms, negative aftereffects occurred (i.e., throwing errors in the opposite direction), which indicates that the short-term learning involved a realignment of the relationship between the exploited informational variables and the aiming action (e.g., Redding \& Wallace, 1997; Willingham, 1998). This points to calibration, rather than a shift in the use of informational variables.

A similar process of calibration was proposed by Van Lier et al. (2011) to explain golfers' improvement in directional putting accuracy with increases in skill level. Yet the origin of the perceptual distortion in golf putting may be crucially different from that in the prism studies. In the prisms studies, errors in perceived direction are artificially induced and usually short-lived, whereas the directional errors in golf putting (or in the perceived direction of the perfect aiming line) occur more generally and are relatively persistent (Cuijpers, Kappers \& Koenderink, 2000). This raises the question of whether, indeed, the directional inaccuracies in putting that emerged from this intrinsic bias or dynamics (see Kelso, 1995) are as easily amendable as the artificially induced errors in the prisms studies.

The second purpose of this study was to scrutinize whether calibration of putting direction generalizes to perceptual judgments of the direction of the perfect aiming line and/or vice versa. To test this, we provided two groups of novice golfers who made consistent and systematic directional errors feedback about the direction and magnitude of the error, while they were either practicing golf putting or practicing perceptual judgments. In subsequent posttests and retention tests, we assessed transfer of calibration. Some have argued that transfer of calibration between tasks occurs if, and only if, the tasks are functionally similar - that is, share the same purpose (e.g., Rieser, Pick, Ashmead \& Garing, 1995; Withagen \& 
Michaels, 2002). Yet the empirical evidence is contentious. Martin et al. (1996) found that adaptation to prisms was specific for the throwing limb (i.e., no transfer of calibration occurred from the right to the left hand) and throwing pattern (i.e., no transfer occurred between overhand and underhand throws), despite these tasks being functionally similar. By contrast, Withagen and Michaels $(2002,2004)$ provided support for the functional hypothesis for transfer of calibration in both motor tasks and perceptual tasks. For example, it was shown that calibration of walking (i.e., the relation between moving speed and optical flow field was realigned by having participants walk on a treadmill in a virtual environment) transferred to crawling. Although different limbs are involved, walking and crawling are locomotor tasks with the same functional goal (i.e., to move from one place to another). In a second study, Withagen and Michaels (2004) demonstrated transfer of calibration of rod length perception from the right hand to the left hand. Hence, with respect to the present study, the issue is whether the putting action and the perception of direction can be considered as functionally similar tasks (e.g., both of them entail obtaining information for directional judgments of the perfect aiming line). In this respect, the influential two-visual-system model of Milner and Goodale (1995, 2008); see also Van der Kamp, Rivas, van Doorn \& Savelsbergh, 2008; for a contrasting view, see Franz, Gegenfurtner, Bülthoff \& Fahle, 2000) proposed that the use of information for action (e.g., the control of the orientation of the club head in putting) and its use for perception (e.g., obtaining knowledge on the direction of the perfect aiming line) are functionally distinct and supported by separate neuroanatomical systems. This provides a reason to suspect that transfer of calibration will not occur between the putting action and the perception of direction. The empirical evidence supporting Milner and Goodale's claim of functionally and neurologically dissociated systems for action and perception, however, is largely confined to observations in real time - that is, for tasks performed on the time scale of seconds (but see Gonzalez, Ganel, Whitwell, Morrissey \& Goodale, 2008). Hence, it remains to be seen whether independence also applies to changes on longer time scales of learning.

By contrast, others have argued that perception and action are tightly integrated and use similar information (e.g., Hommel, Müsseler, Aschersleben \& Prinz, 2001; Prinz, 1997, 2006). Within the common-coding theory, this is conceptualized as perception and action sharing common codes or representations. The notion of common codes for perception and action is corroborated by neuropsychological evidence that specialized neural pathways exist (i.e., the mirror neuron system) that respond during both action and perception (e.g., Blakemore \& Frith, 2005; Rizzolatti \& Craighero, 2004). Hence, to the extent that the putting action and the perception of direction are, indeed, tasks that exploit common codes or use the same information, a strong argument can be made that improvements in putting direction following a process of calibration would transfer to the perception of direction and vice versa.

We report two experiments. Experiment 1 sets the stage by examining the circumstances in which directional errors are most likely to occur consistently and reliably. Subsequently, in Experiment 2, these circumstances were exploited to examine whether augmented feedback on the direction of putting and perceptual errors would induce a process of calibration and to examine whether transfer of calibration would occur between putting direction and perception of direction of the perfect aiming line.

\section{Experiment 1}

Obviously, learning can take place only when systematic and consistent errors exist. Previous work has shown that systematic rightward error in perceived direction occurs at the group level (Johnston et al., 2003; Van Lier et al., 2011), but it has also been suggested that inter- and intraindividual differences transpire in the magnitude of the perceptual distortion (e.g., Cuijpers et al., 2000, 2003; Koenderink \& van Doorn, 1998; Koenderink, van Doorn \& Lappin, 2000, 2003). For example, in golf, the exact position of the eyes (i.e., line of sight), relative to the perfect aim line, affected the magnitude of the perceived directional error (Van Lier et al., 2011). The directional errors occurred only when the eyes were positioned next to the ball and above the hands (positions $\mathrm{H} 3$ and H4, Fig. 2), but not when they were directly above the ball (position P, Fig. 2). Van Lier et al. attributed this increase to there being a larger angle between the line of sight and the perpendicular to the plane in which the direction of the perfect aim line was to be judged. However, alternative explanations, such as the absolute distance or lateral distance (see Fig. 2) between the eyes and the ball, cannot be ruled out. Hence, in Experiment 1, we assessed what head (and eye) position resulted in the most consistent and reliable errors in perceived direction for the individual participants by evaluating the effect of varying the angle between the line of sight and the ground plane and the lateral, vertical, and absolute distances between eyes and ball.

Method

Participants Ten right-handed novice golfers (mean age $=$ $21.9 \pm 2.7$ years) with normal or corrected-to-normal vision volunteered to participate in the experiment. They provided informed consent prior to the experiment and were treated in accordance with the local institution's ethical guidelines. 


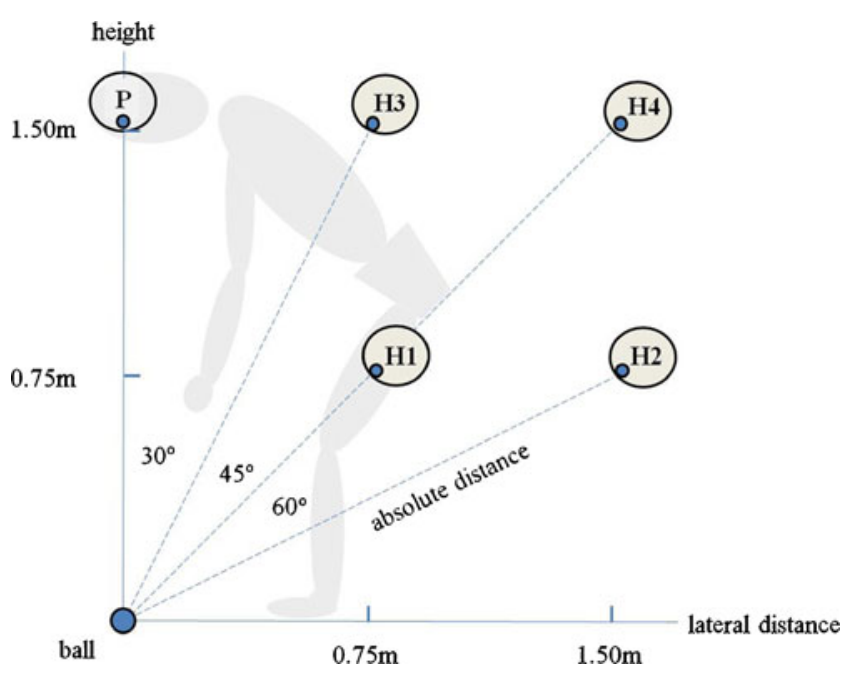

Fig. 2 Schematic representation of the head positions $\mathrm{H} 1$ to $\mathrm{H} 4$ as used in Experiment 1. P indicates the point straight above the ball and perpendicular to the plane in which the direction of the perfect aim line was to be judged

Apparatus The experimental apparatus and procedure was based on earlier work by Van Lier et al. (2011). A triangular-shaped level platform was used that was covered with an artificial grass from synthetic turf (GreenFields, Genemuiden, The Netherlands). The green was approximately $4 \mathrm{~m}$ long and $2 \mathrm{~m}$ wide. The green was cleaned prior to the experiment in order to prevent the presence of any landmarks that might be used as a reference. Additionally, black plastic sheeting, which hung from the ceiling, covered the edges of the green. The sheeting was wrinkled, creating irregular cavities and protrusions so as to minimize any salient reference points for the participants. At a distance of $1.80 \mathrm{~m}$ from the hole, a pointer was placed $2 \mathrm{~cm}$ above the artificial green. The pointer consisted of a golf ball from which a 3-mm-thick needle stuck out $15 \mathrm{~cm}$ from the ball's front and $10 \mathrm{~cm}$ from its back. The pointer could be rotated in a stepwise fashion, using two hand-held switches that fed into a computer. The hand-held switches controlled a servomotor that was connected to the pointer and placed underneath the green. The rotation speed of the pointer was $6^{\circ} / \mathrm{s}$ when the pointer's front was more than $20^{\circ}$ off-target at the time the switch was pressed but was reduced to $1 \%$ shen it was within $20^{\circ}$ off the target. The precision of the pointer was $0.06^{\circ}$.

To consistently vary the position of the head and eyes in relation to the pointer, an adjustable head support was used, which consisted of an adjustable stand bearing a small wooden ball (i.e., $3 \mathrm{~cm}$ in diameter). The participants were instructed to keep the back of their head to the adjustable wooden ball, thereby creating four head and eye positions that varied in height (i.e., at 75 and $150 \mathrm{~cm}$ ) and lateral distance (i.e., 75 and $150 \mathrm{~cm}$ ) relative to the pointer (Fig. 2). Alongside, the angle between the line of sight and the perpendicular to the plane in which the directional judgment was made (i.e., $30^{\circ}, 45^{\circ}, 60^{\circ}$ ) and the absolute distance between the eyes and the pointer (i.e., 106, 168, $212 \mathrm{~cm}$ ) were varied.

Procedure and design The participants stood to the left side of the ball and were instructed, prior to each trial, to contact the head support with the back of their head (i.e., protuberantia occipitalis externa). During the trial, they were allowed to move their head freely (i.e., to look from the pointer to the hole and vice versa) but had to keep their head close to the head support. For the two low head positions (i.e., positions $\mathrm{H} 1$ and $\mathrm{H} 2$ ), participants rested on their knees, while for the two high positions (i.e., positions $\mathrm{H} 3$ and H4), they stood as they would do when addressing a ball in order to putt.

At the start of each trial, the pointer was automatically placed in a random orientation between $30^{\circ}$ and $60^{\circ}$ to either the right or left of the hole. This was changed from trial to trial and prevented participants from making judgments relative to the initial pointer orientation on the current trial and/or the final pointer orientation on the previous trial. Participants were first instructed on how to rotate the pointer by pressing the two hand-held switches. Pressing the switch in the left hand resulted in the pointer's rotating in a clockwise direction, while pressing the switch in the right hand made the pointer rotate in a counterclockwise direction. Participants were then instructed to rotate the pointer such that it pointed to the center of the hole, which was indicated by the foot of a flagpole. The pointer's exact orientation with respect to the perfect aiming line was registered by a computer once the participants verbally indicated that they had positioned the pointer correctly.

The four head position conditions were administered in blocks of 12 trials in a counterbalanced order across participants. Participants did not receive any knowledge of results during the experiment.

Data reduction and statistics The error in perceived direction served as the dependent variable. It was defined as the angle between the direction of the pointer and the direction of the true line between the ball and the hole (i.e., perfect aiming line). A negative angle indicated a counterclockwise error (i.e., leftward error), whereas a positive angle indicated a clockwise error (i.e., rightward error). To test whether the individual participants made consistent and systematic errors in perceived direction, we used one-sample $t$-tests with the Šidák correction for multiple comparisons to assess whether the error in perceived direction was different from zero (i.e., no error) for each participant and head position separately. In addition, individual regression analyses were conducted to examine which factor or combination of factors contributed most to the directional error. 


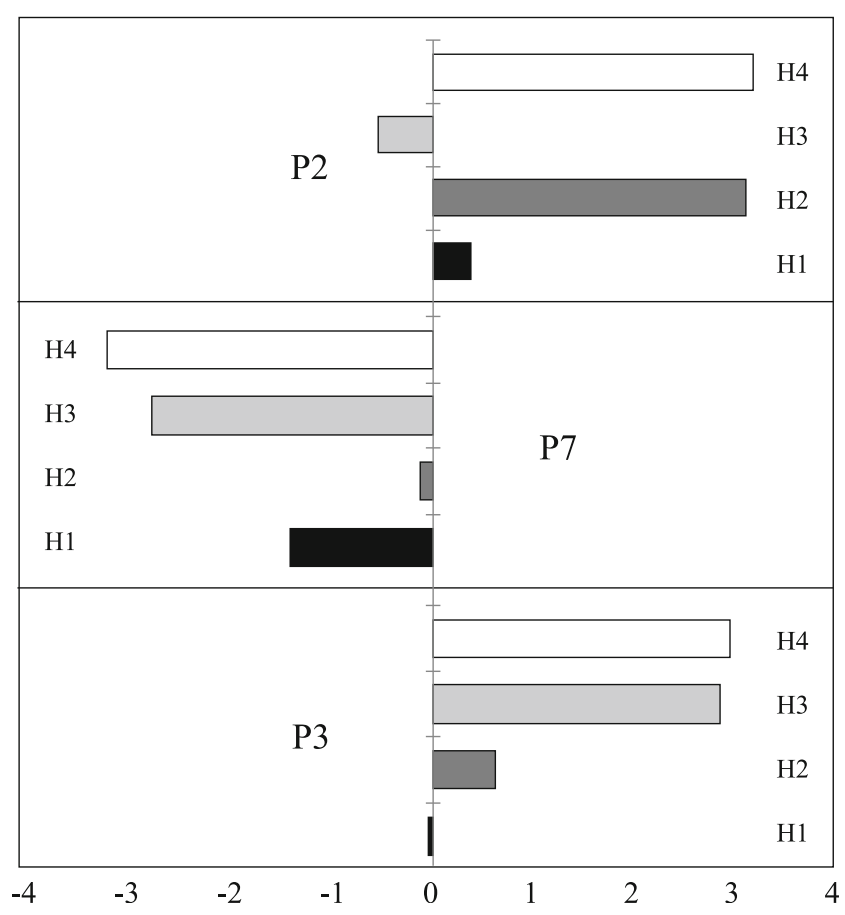

Fig. 3 Errors in perceived direction as a function of head positions $\mathrm{H} 1$ to $\mathrm{H} 4$ for 3 individual participants (P2, P7, P3) in Experiment 1. Positive and negative errors indicate leftward and rightward errors, respectively

\section{Results and discussion}

Figure 3 shows the pattern of errors in perceived direction for 3 individual participants. The interindividual differences immediately stand out. For example, participant P3 shows larger errors for head positions at larger heights (i.e., H3 and H4). This is also the case for participant P7, but with the errors in the opposite direction (i.e., leftward instead of rightward errors). For participant P5, however, the error in perceived direction was chiefly affected by lateral distance, with larger rightward errors for head positions $\mathrm{H} 2$ and $\mathrm{H} 4$. Notwithstanding these interindividual differences, $t$-tests revealed that errors in perceived direction most consistently and reliably occurred for head position H4. That is, 8 participants showed a significant error, 7 of whom made the anticipated rightward error, $t \mathrm{~s}(11)>3.2, p \mathrm{~s}<.01$, while 1 participant made a significant error to the left of the hole, $t(11)=15.7, p<.01$. The 1 final participant did not produce a significant error for head position $\mathrm{H} 4, t(11)=1.9, p>.05$. Three to 5 participants made significant errors in perceived direction for the other three head positions. The source of the interindividual differences remains uncertain; the regression analysis outcomes point to the individual participants' errors in perceived direction being related differently to angle between the line of sight and the perpendicular to the plane in which the pointer was rotated and the vertical, lateral, and absolute distances between the eyes and the pointer (Table 1). Previous work had pointed to the angle between the line of sight and the perpendicular to the plane in which the judgment was made as the most important determinant for the size of the error in perceived direction (Van Lier et al., 2011). However, none of the individual patterns of error was in line with this suggestion. Finally, we conclude that the largest combined vertical and lateral distance between the eyes and the ball (i.e., head position H4) most reliably resulted in errors in perceived direction (although not completely consistent across participants). We therefore used this head position in Experiment 2 to assess learning.

\section{Experiment 2}

Experiment 2 investigated whether augmented outcome feedback induces relatively permanent changes in putting and perception. Previous work has shown that outcome feedback (or knowledge of results) on the magnitude and sign of error aids the learner in enhancing calibration of an informational variable to a task variable (i.e., $I_{(\mathrm{t})}$ and $T_{(\mathrm{t})}$ in Eq. 1; see, e.g., Cabe \& Wagman, 2010; Wagman, McBride \& Trefzger, 2008; Withagen \& Michaels, 2005). Wagman et al. (see also Gibson \& Bergman, 1954) argued that such calibration is revealed in increases in accuracy and consistency of performance. In a series of length perception studies, it was found that improvements in the accuracy of performance (i.e., as indicated by a change in constant error) were conditional upon the presence of outcome feedback. By contrast, improvements in the consistency or variability of performance (i.e., as indicated by a change in variable error) occurred irrespective of the presence of feedback; only a few repetitions without feedback apparently sufficed.

Table 1 Outcomes for the regression analysis for the individual participants, displaying the variable that entered the regression equation and the corresponding coefficient

\begin{tabular}{lll}
\hline Participant & Variables Entered & Beta Coefficients \\
\hline P1 & height & 0.52 \\
P2 & absolute distance & 0.59 \\
P3 & height & 0.51 \\
P4 & none & - \\
P5 & lateral distance & 0.71 \\
P6 & lateral distance & 0.73 \\
P7 & height & -0.61 \\
P8 & lateral distance & 0.71 \\
P9 & absolute distance & -0.41 \\
P10 & none & - \\
\hline
\end{tabular}


In the present study, two groups of novice golfers received outcome feedback on the magnitude and sign of the directional errors they made while practicing either putting or perception with their head positioned at a large vertical and lateral distance from the ball (i.e., position $\mathrm{H} 4$ per Experiment 1). A pretest, practice, posttest, and retention test design followed. We hypothesized that outcome feedback induces calibration, which would result in enhanced performance accuracy and increased consistency (i.e., reduced variability) at the posttest and retention test, relative to the pretest. Our chief interest, however, was in whether calibration induced by putting practice with outcome feedback would transfer to perception and, vice versa, whether calibration due to perception training with feedback would transfer to putting. We expected transfer of calibration to occur to the degree that the tasks had functional characteristics in common (e.g., they crucially depended on accurate information, or codes, about the direction of perfect aiming line; see Hommel et al., 2001; Prinz, 1997; see also Van Lier et al., 2011). We expected the transfer to be restricted to performance accuracy and not to comprise consistency, because an increase in consistency requires at least a limited amount of repetitions. Alternatively, however, following Milner and Goodale (1995, 2008; see also Withagen \& Michaels, 2004), the putting action and perception task may be considered as functionally separate, which would rule out the possibility that transfer of calibration between the tasks would occur.

\section{Method}

Participants Thirty-nine right-handed novice golfers with normal or corrected-to-normal vision volunteered to participate. In order to ensure that participants were unskilled, nine volunteers were excluded from further participation in the experiment because they sunk more than four out of ten putts during the pretest (see the Procedure section below). Five more participants were excluded after completion of the experiment because the average constant errors in the putting and/or perception task during the pretest did not exceed zero. Finally, due to technical failure, the data for 1 participant were lost. The remaining participants were randomly assigned to the perception-training group $(n=9$; mean age $=22.2 \pm 2.8$ years), the action-training group ( $n=8$; mean age $=23.4 \pm 3.0$ years $)$, and the control group ( $n=8$; mean age $=25.0 \pm 4.6$ years $)$. The volunteers received a small monetary fee and were treated in accordance with the local institution's ethical guidelines.

Materials and apparatus For the perception task, the same pointer was used as per Experiment 1. The putting task was performed on the same platform, which was prepared prior to testing to ensure that the ball roll was unaffected (i.e., a speed of 14 Stimp). Using standard golf balls, participants had to perform putts using a specially fitted long conventional putter. The length $(1.21 \mathrm{~m})$ and lie angle $\left(50^{\circ}\right)$ of the putter allowed one to make putts comfortably while standing with the head positioned at $1.50 \mathrm{~m}$ above and next to the ball (i.e., position H4 in Experiment 1). The adjustable head support (see Experiment 1) was used to assist participants in maintaining this position.

Liquid crystal goggles (Plato Translucent Technologies, Toronto, Canada) were used to remove visual feedback about task outcome. During the perception task, the goggles turned opaque after the participants indicated that they had rotated the pointer in the desired position, and in the putting task, the goggles turned opaque the moment the ball interrupted a light beam of a photoelectric switch (Omron E3S-R 30E4), which was positioned perpendicular to the ball path at $40 \mathrm{~cm}$ from the initial ball position. The hole was covered with a white artificial grass plug to eliminate auditory feedback from the ball entering the hole.

Two digital video cameras (Panasonic $25 \mathrm{~Hz}$ interlaced PAL) were used. One camera was positioned directly above the ball and was used to determine the initial direction of the ball roll. The second camera with a transparent visor was placed behind the ball, directly in line with the ballhole direction. Its recordings were displayed on a monitor to provide verbal feedback about the distance the ball passed next to the hole during the putting task (i.e., this reflects the magnitude and sign of the directional error). To this end, a foot-rule was drawn on the monitor in such a manner that it precisely overlapped the line through the center of the hole that was perpendicular to the perfect aim line. The foot-rule indicated intervals corresponding to $5 \mathrm{~cm}$ on the platform.

Procedure and design Participants were randomly assigned to one of three groups on the basis of their pretest performance. The perception- and action-training groups followed a pretest, practice, posttest, retention test design. The control group, however, did not receive practice. Participants first performed the pretest, which was followed by three practice sessions that took place at separate days. The posttest was performed on the same day as the third practice session, with a 15 -min break in between. Finally, the retention test was conducted between 2 and 4 days after the posttest.

During the pretest, posttest, and retention test, the participants performed a block of 12 perception trials and a block of 12 putting trials, the order of which was counterbalanced across participants. The tests were administered in two bouts of 6 trials. The perception task was similar to that in in Experiment 1. That is, the participants rotated the pointer such that it pointed to the center of the hole. In the putting task, participants attempted to putt the 
ball into the hole. Participants wore the Liquid Crystal goggles to ensure that visual feedback of the task outcome could not be used to improve performance on the subsequent trial. In the perception task, the glasses turned opaque after the participants indicated that they had rotated the pointer in the desired position. In the putting task, the goggles turned opaque at the moment the ball had traveled $40 \mathrm{~cm}$ in the direction of the hole. Participants did not receive augmented feedback during the test trials. Participants were constrained to consistently position the head at a height of $150 \mathrm{~cm}$ and a lateral distance of $150 \mathrm{~cm}$, relative to the pointer or the ball (i.e., head position $\mathrm{H} 4$ in Experiment 1). Although they were allowed to move their head in order to look from the pointer or the ball to the hole and vice versa, they had to keep it as close as possible to the head support. After completion of a trial (i.e., after the glasses were closed), the participant turned his or her back to the hole until the experimenter opened the goggles. This was the sign to prepare for the next trial.

During each of the three practice sessions, the participants in the action-training group performed 48 putting trials, while the participants in the perception-training group performed 48 perception trials. These sessions were administered in bouts of 6 trials, in between which participants were allowed to take short rests to prevent fatigue and to relax. The procedure was similar to that in the pretest, posttest, and retention test, but the participants now received verbal augmented feedback on the magnitude and sign of the directional error after each trial. Feedback was provided in intervals of $5 \mathrm{~cm}$ distance to the left or right from the center of the hole. To this end, the directional error in degrees for the perception task was directly converted into distances from the hole's center. Specifically, (1) when the pointer was rotated or the ball passed within $5 \mathrm{~cm}$ of the center of the hole, participants were told that it pointed to or passed "in the hole to the left or right from the center"; (2) when the pointer pointed to or the ball passed the hole within $30 \mathrm{~cm}$ of its center, participants were told that it pointed or passed the corresponding $5 \mathrm{~cm}$ interval to the left or right from the center of the hole; (3) when the pointer pointed to or the ball passed more than $30 \mathrm{~cm}$ from the centr of the hole, the participants were told "more than $30 \mathrm{~cm}$ to the left or right from the center." Before each practice session, participants were briefly informed of the feedback procedure.

Data reduction and statistics The error in perceived direction (in degrees) was defined as the angle between the direction of the pointer and the direction of the true line between the ball and the hole (i.e., perfect aim line). A custom-made semiautomatic video-analyzing program, developed with the Matlab ${ }^{\circledR}$ Image Processing Toolbox, was used to digitalize the path of the ball and its direction for the first ten frames (i.e., $400 \mathrm{~ms}$ ) after contact. The error in putt direction was defined as the angle between the direction of ball roll and the true line between the ball and hole. Negative angles for the errors in perceived and putting direction indicated a leftward or counterclockwise error, while positive angles indicated a rightward or clockwise error.

As in Experiment 1, perusal of the pretest data showed that most participants made reliable and consistent rightward errors in both the perception and putting tasks. Yet one participant made consistent leftward errors in both tasks, whereas a second participant made leftward errors in the perception task and rightward errors in the putting task. To make sure that these interindividual differences in the sign of the directional error and the changes therein as a function of practice did not cancel each other out, the errors in the pretest were transformed into a rightward error (i.e., positive sign). In addition, the errors during practice and in the posttest and retention test were adjusted such that the magnitude and the sign of the differences with the pretest were maintained.

Subsequently, we submitted constant errors (i.e., accuracy) and variable errors (i.e., consistency) in perceived direction and putting direction to separate 3 (group: perception training, action training, control) $\times 3$ (test: pretest, posttest, retention test) ANOVAs with repeated measures on the last factor. Huyn-Feldt corrections to the degrees of freedom were applied in the case of any violations of sphericity, and partial eta-squared $\left(\eta_{\mathrm{p}}{ }^{2}\right)$ values were computed to determine the proportion of total variability attributable to each factor or combination of factors. Post hoc comparisons were conducted using Tukey's HSD test $(p<.05)$. One-sample $t$-tests with the Šidák correction for multiple comparisons were used to examine whether the constant errors were significantly larger than zero (i.e., no error).

Finally, the accuracy and consistency during perception training and action training were calculated in 12 blocks of 12 trials. This was based on the $5-\mathrm{cm}$ distance interval feedback that was provided to the participants. The constant error (i.e., accuracy) and variable error (i.e., consistency) in perceived direction for the perception-training group and the putting direction for action-training group were submitted to a 12 (block: 1 to 12) ANOVA with repeated measures. Additionally, two-tailed one-sample $t$-tests were used to assess whether the constant directional errors were larger than zero (i.e., no error).

Results and discussion

Figures 4 and 5 illustrate the constant and variable errors in the pretest, posttest, and retention test for the putting task 


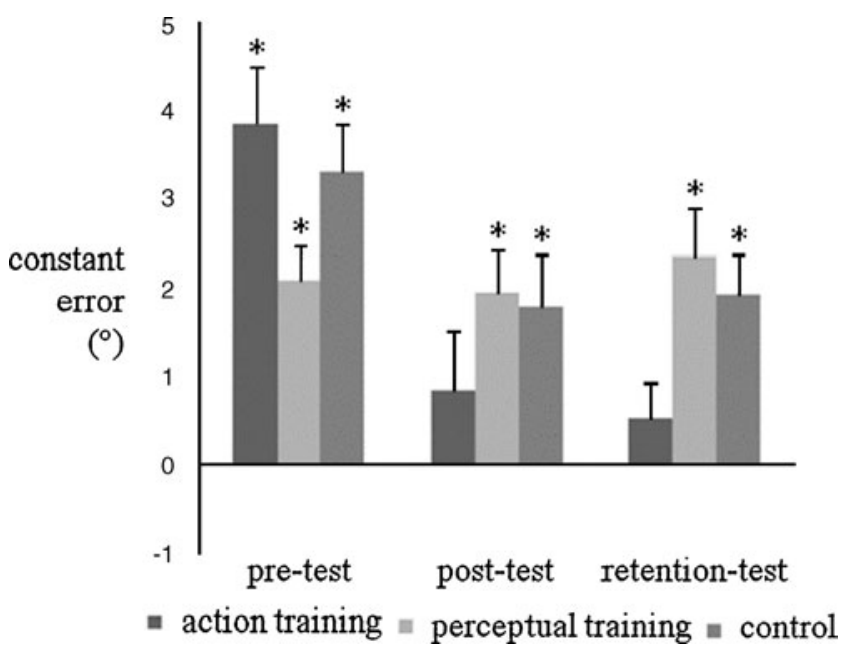

Fig. 4 Constant errors in putting for the pre-, post-, and retention tests. Error bars indicate variable error, and asterisks indicate errors that are significantly different from zero $\left({ }^{*} p<.05\right)$

and the pointing task, respectively. Figure 4 shows that putting accuracy (i.e., the constant error) was reduced only for the action-training group and that this increase in accuracy was maintained during retention. This was confirmed with significant effects for test, $F(2,44)=$ $11.85, p<.001, \eta_{\mathrm{p}}{ }^{2}=.35$, and the test $\times$ group interaction, $F(4,44)=4.82, p<.05, \eta_{\mathrm{p}}{ }^{2}=.31$, for the constant putting error. The effect of group was not significant, $F(2,22)=$ 0.52. Post hoc comparisons indicated that the directional errors in the posttest and retention test were significantly smaller than in the pretest, but this decrease occurred only for the action-training group. In addition, $t$-tests showed that the directional errors were significantly larger than zero, except for the errors of the action-training group in the

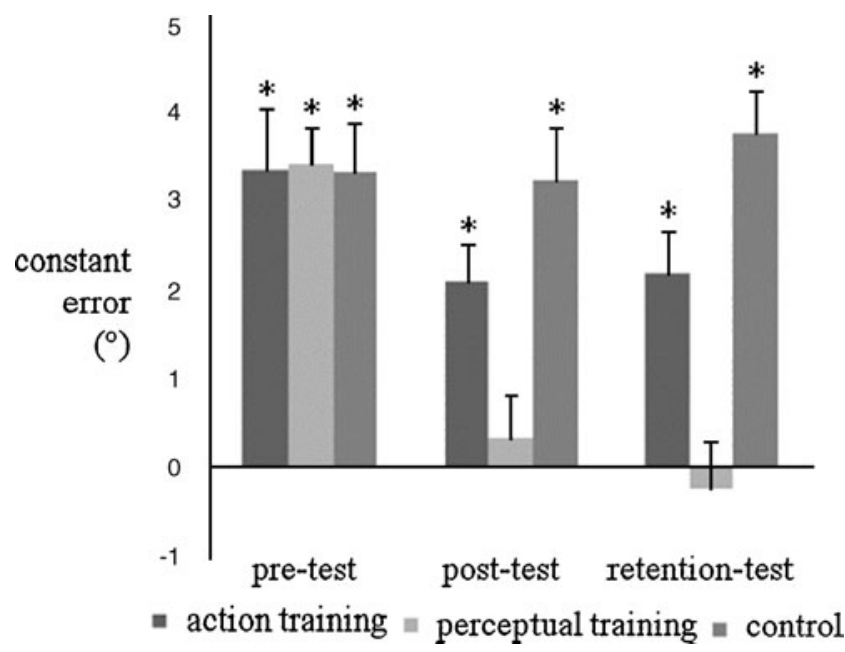

Fig. 5 Constant errors in perceived direction for the pre-, post-, and retention tests. Error bars indicate variable error, and asterisks indicate errors that are significantly different from zero $\left({ }^{*} p<.05\right)$ posttest and retention test, $t \mathrm{~s}<2.0, p \mathrm{~s}>.08$. A subsequent ANOVA on the variable directional errors revealed no significant increase in consistency of putting, although the effect of test was nearly significant, $F(2,44)=$ $3.03, p=.07, \eta_{\mathrm{p}}{ }^{2}=.12$.

Figure 5 shows a similar pattern of findings for the perception task. Thus, significant effects for test, $F(2,44)=$ $7.00, p<.05, \eta_{\mathrm{p}}{ }^{2}=.24$, group, $F(2,22)=7.31, p<.05, \eta_{\mathrm{p}}{ }^{2}=$ .40 , and the test $\times$ group interaction, $F(4,44)=3.88, p<.05$, $\eta_{\mathrm{p}}{ }^{2}=.26$, were found for the constant perception error. Post hoc comparisons indicated that the directional error of the perception-training group was significantly smaller in the posttest and retention test, in comparison with the pretest. In addition, $t$-tests showed that perception errors were significantly different from zero, with the exception of the errors of the perception-training group in the posttest and retention test, $t \mathrm{~s}<.81, p \mathrm{~s}>.44$. In sum, with practice, only the perception training had reduced the perceptual error to zero. The ANOVA for the variable error revealed that consistency of the directional judgments was significantly affected by test only, $F(2,44)=6.01, p<.01, \eta_{\mathrm{p}}{ }^{2}=.22$. Post hoc comparisons indicated that variable error was significantly reduced in the retention test, as compared with the pretest.

Finally, Figs. 6 and 7 depict the changes in accuracy and consistency of the action-training and perception-training groups, respectively. They show that the largest performance gains were achieved during the initial training blocks, which is suggestive of a typical exponential learning curve. This was confirmed by significant effects of block for putting error, $F(11,66)=3.10, p<.01, \eta_{\mathrm{p}}{ }^{2}=$ .34 , and perception error, $F(11,88)=2.47, p<.05, \eta_{\mathrm{p}}{ }^{2}=$ .24. Post hoc comparisons indeed indicated that the constant errors were significantly larger in the first two blocks, as compared with the two final practice blocks. In addition, two-tailed one-sample $t$-tests showed that from

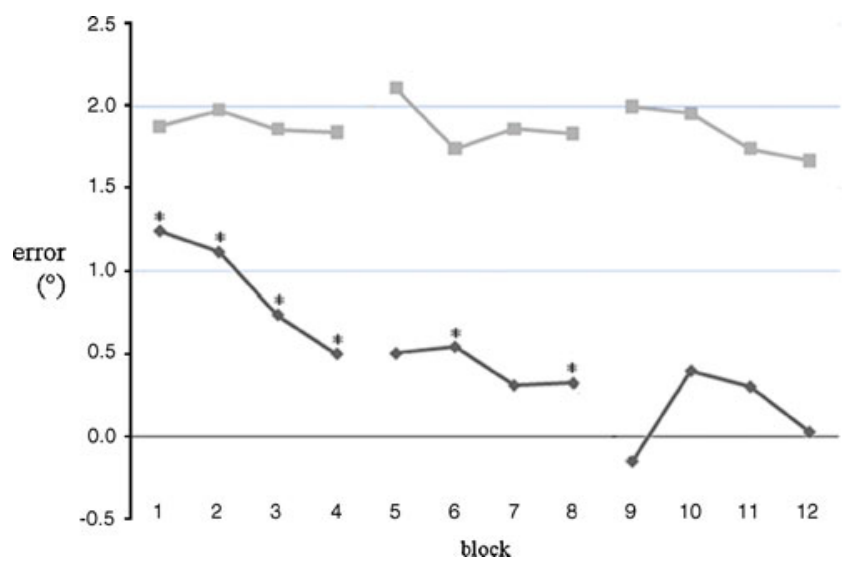

Fig. 6 Constant error (black line) and variable error (gray line) in putting as a function of blocks of 12 trials during practice. Asterisks indicate errors that are different from zero $\left({ }^{*} p<.05\right)$ 


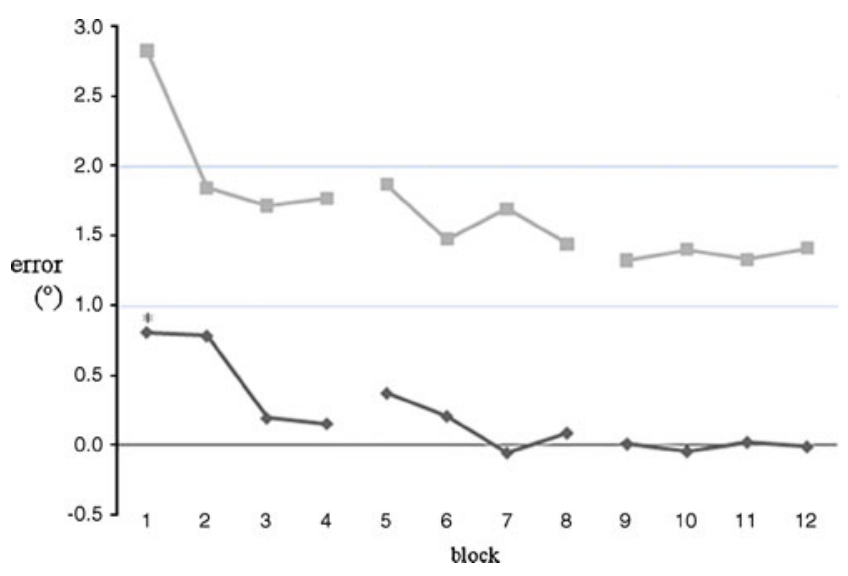

Fig. 7 Constant error (black line) and variable error (gray line) in perceived direction as a function of blocks of 12 trials during practice. Asterisks indicate errors that are different from zero $\left({ }^{*} p<.05\right)$

block 9 , putting errors were not significantly different from zero (i.e., no error), whereas for the perception task, this occurred from block 6 onward. Finally, Figs. 6 and 7 suggest that practice did increase consistency of perception but, perhaps surprisingly, did not result in more consistent putting. Accordingly, only for the perception-training group was a significant effect of block found for the variable error, $F(11,88)=4.94, p<.01, \eta_{\mathrm{p}}{ }^{2}=.38$. Post hoc comparisons indicated that this decrease in variable error occurred from the first to the second block.

\section{General discussion}

A first purpose of the present study was to examine whether improvements induced by outcome feedback about the size and sign of the directional error in golf putting are driven by calibration. Indeed, the reduction of the initial putting errors to zero after putting practice with feedback strongly points to calibration. This is further underlined by the gradual increase in accuracy (i.e., decrease in constant error) during practice. It must be acknowledged that although the findings are consistent with calibration, the present design does not conclusively demonstrate that calibration occurred. A genuine demonstration of a rescaling between information and task variables (i.e., presumably, for putting, this task variable is the orientation of the club head at impact) requires that the informational variable is varied (e.g., by manipulating distance between the ball and hole and/or the head position relative to the ball). Nevertheless, the results show that this putative calibration was relatively permanent; that is, putting accuracy was maintained on a 1-week retention test. As far as we know, and perhaps somewhat surprisingly, this is the first study framed within an ecological theory of learning to investigate and suggest that calibration can lead to relatively permanent changes between informational and task variables (cf. Withagen \& Caljouw, 2011). It is perhaps illustrative to compare the present interpretation with that in a recent report by Cavina-Pratesi, Kuhn, Ietswaart and Milner (2011). These authors investigated real and pantomimed reaching movements of magicians. Unlike nonmagicians, for whom pronounced kinematic differences were observed between real and pantomime grasping, among magicians the kinematics of the two types of grasping were nearly identical. The authors suggested that with sustained practice the magicians had recalibrated the control of the reaching movements from information of the real object toward information from a spatially separate location. An analogous interpretation for the present findings would be that the outcome feedback during putting practice led the participant to aim for a "ghost" hole next to the real hole, as if the golfer compensated for perceptual distortion. Instead, we argue that the relation between the visual information and the putting action is adjusted, rather than corrections being made for a misperception of the location of the target (see also Van Lier et al., 2011). This is supported by the observation that improvements in putting accuracy are not contingent upon improvements in perception of the direction of the perfect aiming line.

The findings with regard to the perception of the direction of the perfect aiming line were similar, although the increase in perceptual accuracy during practice seemed to occur much more rapidly than for putting. A more notable distinction between the putting and perception tasks is related to the consistency or variability of performance. For the perception task, consistency increased very rapidly after 10 to 20 trials only. Also, in line with earlier observations for length and distance perception (e.g., Gibson \& Bergman, 1954; Wagman et al., 2008; Withagen $\&$ Michaels, 2004), these increases in consistency seem not to have depended on feedback, since increases were not restricted to the perception-training group, but also became apparent in the retention test for the action-training and control groups. By contrast, for the action task, changes in consistency were observed neither from the pretest to the posttest or retention test nor during practice. It is not particularly clear why, but one suggestion may be that standardization of movement occurs relatively late during the learning of action. For example, Koedijker et al. (2011) have recently proposed that automatization (i.e., as indicated by reliance of movement execution on working memory) of a table tennis forehand stroke transpired before standardization of the stroke. Irrespective of whether this proposal is correct, the divergent patterns for consistency suggest that calibrations for the putting and the perception tasks were not identical.

The main purpose, however, was to examine transfer of calibration. Perception training resulted in enhanced perception of direction but did not reduce the directional errors 
in putting. And conversely, action training did increase putting accuracy but did not lead to reliable improvements in the perception of direction of the perfect aiming line. In other words, we found no evidence that transfer of calibration between action and perception occurred. Nor was there evidence to support the contention of transfer of calibration with respect to consistency or variability of performance. The action training, however, did result in an increased consistency of the perceptual judgments, but this cannot be attributed to the training, since the control group showed a similar increase in consistency. These findings are in line with the proposal of the existence of functionally distinct systems for the use of information in action and perception (Milner \& Goodale, 1995, 2008) but are much more difficult to reconcile with ideas of a common informational, or representational, basis for action and perception (Hommel et al., 2001: Prinz, 1997, 2006). The putting action and the perception of direction are best understood as giving rise to separate couplings between task and informational variables. These couplings may entail not only distinct task variables (i.e., $T_{(\mathrm{t})}$ ), but also distinct informational variables (i.e., $\left.I_{(\mathrm{t})}\right)$, even though both tasks critically depend on information about the direction of the perfect aiming line. Thus, Van Lier et al. (2011); see also Johnston et al., 2003) demonstrated that the perception of direction of the aiming line (see Experiment 1 above) was a function of head-eye position with respect to the ball, while directional error in putting accuracy was immune to differences in head-eye position. This indicates the use of different informational variables in the control of the putting action and the perception of direction. If the calibration or scaling of an informational variable to a task variable is unique to a particular coupling, transfer of calibration between the two tasks is unlikely.

The absence of transfer is contrary to previous suggestions by Van Lier et al. (2011). They observed that expert golfers, relative to novice golfers, tended to produce leftward errors in the perception of the perfect aiming line (see Fig. 1). Because the novice players made rightward errors in putting, it was argued that the calibration of the putting action induced by practice would have transferred to the perception of direction of the aiming line. The present findings, however, did not reliably show this parallel leftward shift in perception. Yet the novice participants' practice in the present study was limited to only 144 putts over 3 days. This strongly contrasts to expert golfers who have made thousands of putts over at least 10 years. Clearly, we cannot rule out the possibility that over longer time scales of months or years, transfer may occur. In this respect, it is notable that numerically (but not statistically!), there is a trend for the action-training group, and not for the control group, to make more accurate perceptual judgments in the posttest and retention test than in the pretest, while this was not the case for the control group.
Visual perception plays an indispensable role in a large variety of sports skills, including aiming skills, such as putting in golf, and interception skills, such as hitting a ball in tennis or saving a penalty kick in soccer, and so forth. In recent years, a number of studies have attempted to improve these skills through perceptual learning interventions (e.g., Rowe \& McKenna, 2001; Savelsbergh et al., 2010; Smeeton, Williams, Hodges \& Ward 2005; Williams, Ward, \& Chapman, 2003). Typically, researchers do this by repeatedly displaying video clips that represent the situation of interest (e.g., a penalty kicker in the run-up to the ball). By providing instruction or feedback about the pertinent sources of information in the display (e.g., directing attention to the placement of the nonkicking foot), researchers try to enhance a player's perception. Yet these interventions have resulted in mixed success. Despite improvements in perception for the training task, transfer to the actual action has often remained unclear. One concern that has been raised is the degree to which the information available in the display is impoverished (e.g., Abernethy, Thomas \& Thomas, 1993; Dicks, Davids \& Button, 2009). In the present study, however, the perceptual-training environment was not recreated by using 2-D displays but was identical to the real-life performance situation. In all likelihood, a more crucial issue with these practice interventions is, therefore, that perceptual learning takes place independently of action. Whereas the use of information for perception is facilitated, it leaves the intricate coupling between information and movement untouched (Dicks, Button \& Davids, 2010; Milner \& Goodale, 2008). Perception for action cannot be trained in isolation from action (see also Van der Kamp et al., 2008).

To conclude, we have shown that directional errors in golf putting can be permanently canceled on basis of outcome feedback, consistent with a process of calibration. Similarly, outcome feedback can also annihilate errors in the perception of the perfect aiming line. Yet the observed increases in accuracy were specific to the trained task. Thus, no transfer of calibration occurred between perception and action.

Open Access This article is distributed under the terms of the Creative Commons Attribution Noncommercial License which permits any noncommercial use, distribution, and reproduction in any medium, provided the original author(s) and source are credited.

\section{References}

Abernethy, B., Thomas, K. T., \& Thomas, J. T. (1993). Strategies for improving understanding of motor expertise (or mistakes we have made and things we have learned!). In J. Starkes \& F. Allard (Eds.), Cognitive issues in motor expertise (pp 317-358). New York: North-Holland. 
Blakemore, S. J., \& Frith, C. (2005). The role of motor contagion in the prediction of action. Neuropsychologia, 43, 260-267.

Cabe, P. A., \& Wagman, J. B. (2010). Characterizing perceptual learning using regression statistics: Development of a perceptual calibration index. The American Journal of Psychology, 123, 253-267.

Cavina-Pratesi, C., Kuhn, G., Ietswaart, M., \& Milner, A. D. (2011). The magic grasp: Motor expertise in deception. PlosONE, 6, 1-5.

Cuijpers, R. H., Kappers, A. M. L., \& Koenderink, J. J. (2000). Investigation of visual space using an exocentric pointing task. Perception \& Psychophysics, 62, 1556-1571.

Cuijpers, R. H., Kappers, A. M. L., \& Koenderink, J. J. (2003). The metrics of visual and haptic space based on parallelity judgements. Journal of Mathematical Psychology, 47, 278-291.

Dicks, M., Button, C., \& Davids, K. (2010). Examination of gaze behaviors under in situ and video simulation task constraints reveals differences in information pickup for perception and action. Attention, Perception, \& Psychophysics, 72, 706-720.

Dicks, M., Davids, K., \& Button, C. (2009). Representative design for the study of perception and action in sport. International Journal of Sport Psychology, 40, 506-524.

Ernst, M. O., \& Banks, M. S. (2002). Humans integrate visual and haptic information in a statistically optimal fashion. Nature, 415, 429-433.

Franz, V. H., Gegenfurtner, K. R., Bülthoff, H. H., \& Fahle, M. (2000). Grasping visual illusions: No evidence for a dissociation between perception and action. Psychological Science, 11, 20-25.

Gibson, E. J., \& Bergman, R. (1954). The effect of training on absolute estimation of distance over the ground. Journal of Experimental Psychology, 48, 473-482.

Gonzalez, C. L. R., Ganel, T., Whitwell, R. L., Morrissey, B., \& Goodale, M. A. (2008). Practice makes perfect, but only with the right hand: Sensitivity to perceptual illusions with awkward grasps decreases with practice in the right but not the left hand. Neuropsychologia, 46, 624-631.

Hommel, B., Müsseler, J., Aschersleben, G., \& Prinz, W. (2001). The theory of event coding (TEC): A framework for perception and action planning. The Behavioral and Brain Sciences, 24, 849-937.

Jacobs, D. M., \& Michaels, C. F. (2007). Direct learning. Ecological Psychology, 19, 321-349.

Johnston, A., Benton, C. P., \& Nishida, S. (2003). Golfers may have to overcome a persistent visuospatial illusion. Perception, 32, 11511154.

Kelso, A. S. (1995). Dynamic patterns: The self-organization of brain and behavior. Cambridge: MIT Press.

Koedijker, J. M., Poolton, J. M., Maxwell, J. P., Oudejans, R. D. D., Beek, P. J., \& Masters, R. S. W. (2011). Attention and time constraints in perceptual-motor learning and performance: Instruction, analogy, and skill level. Consciousness and Cognition, 20, 245-256.

Koenderink, J. J., \& van Doorn, A. J. (1998). Exocentric pointing. In L. R. Harris \& M. Jenkin (Eds.), Vision and action (pp. 295313). Cambridge: Cambridge University Press.

Koenderink, J. J., van Doorn, A. J., \& Lappin, J. S. (2000). Direct measurement of curvature of visual space. Perception, 29, 69-79.

Koenderink, J. J., van Doorn, A. J., \& Lappin, J. S. (2003). Exocentric pointing to opposite targets. Acta Psychologica, 112, 71-87.

Martin, T. A., Keating, J. G., Goodkin, H. P., Bastian, A. J., \& Thach, W. T. (1996). Throwing while looking through prisms: I. Focal olivocerebellar lesions impair adaptation. Brain, 119, 1183-1198.

Milner, A. D., \& Goodale, M. A. (1995). The visual brain in action. Oxford: Oxford University Press.

Milner, A. D., \& Goodale, M. A. (2008). Two visual systems reviewed. Neuropsychologia, 46, 774-785.
Prinz, W. (1997). Perception and action planning. European Journal of Cognitive Psychology, 9, 129-154.

Prinz, W. (2006). What re-enactment earns us. Cortex, 42, 515-517.

Redding, G. M., \& Wallace, B. (1997). Adaptive spatial alignment. Hillsdale: Erlbaum.

Rieser, J. J., Pick, H. L., Ashmead, D. H., \& Garing, A. E. (1995). Calibration of human locomotion and models of perceptualmotor organization. Journal of Experimental Psychology. Human Perception and Performance, 21, 480-497.

Rizzolatti, G., \& Craighero, L. (2004). The mirror neuron system. Annual Review of Neurosciences, 27, 169-192.

Roberts, R., \& Turnbull, O. H. (2010). Putts that get missed on the right: Investigating lateralized attentional biases and the nature of putting errors in golf. Journal of Sport Sciences, 28, 369-374.

Rowe, R. M., \& McKenna, F. P. (2001). Skilled anticipation in realworld tasks: Measurements of attentional demands in the domain of tennis. Journal of Experimental Psychology: Applied, 7, 60-67.

Savelsbergh, G.J.P., van Gastel, P.J., \& van Kampen, P.M. (2010) Anticipation of penalty kicking direction can be improved by directing attention through perceptual learning. International Journal of Sport Psychology, 41, 24-41.

Smeeton, N.J., Williams, A.M., Hodges, N.J., \& Ward, P. (2005). The relative effectiveness of various instructional approaches in developing anticipation skill. Journal of Experimental Psychology: Applied, 11, 98-110.

Van der Kamp, J., Oudejans, R. D. D., \& Savelsbergh, G. J. P. (2003). The development and learning of the visual control of movement: An ecological perspective. Infant Behavior \& Development, 26, 495-515.

Van der Kamp, J., Rivas, F., van Doorn, H., \& Savelsbergh, G. J. P. (2008). Ventral and dorsal system contributions to visual anticipation in fast ball sports. International Journal of Sport Psychology, 39, 100-130.

Van Lier, W., Van der Kamp, J., \& Savelsbergh, G. J. P. (2011). Perception and action in golf putting: Skill differences reflect calibration. Journal of Sport \& Exercise Psychology, 30(33), 349-369.

Wagman, J. B., McBride, D. M., \& Trefzger, A. J. (2008). Perceptual experience and posttest improvements in perceptual accuracy and consistency. Perception \& Psychophysics, 70, 1060-1067.

Warren, W. H. (1988). The perception-action coupling. In H. Bloch \& B. I. Bertenthal (Eds.), Sensory-motor organizations and development in infancy and early childhood (pp. 23-37). Dordrecht: Kluwer Academic.

Williams, A.M., Ward, P., \& Chapman, C. (2003) Training perceptual skill in field hockey: Is there transfer from the laboratory to the field? Research Quarterly for Exercise and Sport, 74, 98-103.

Willingham, D. B. (1998). A neuropsychological theory of motor skill learning. Psychological Review, 105, 558-584.

Withagen, R., \& Caljouw, S. R. (2011). Aging affects attunement in perceiving length by dynamic touch. Attention, Perception, \& Psychophysics, 73, 1216-1226.

Withagen, R., \& Michaels, C. F. (2002). The calibration of walking transfers to crawling: Are action systems calibrated? Ecological Psychology, 14, 223-234.

Withagen, R., \& Michaels, C. F. (2004). Transfer of calibration in length perception by dynamic touch. Perception \& Psychophysics, 66, 1282-1292.

Withagen, R., \& Michaels, C. F. (2005). The role of feedback information for calibration and attunement in perceiving length by dynamic touch. Journal of Experimental Psychology. Human Perception and Performance, 31, 1379-1390. 